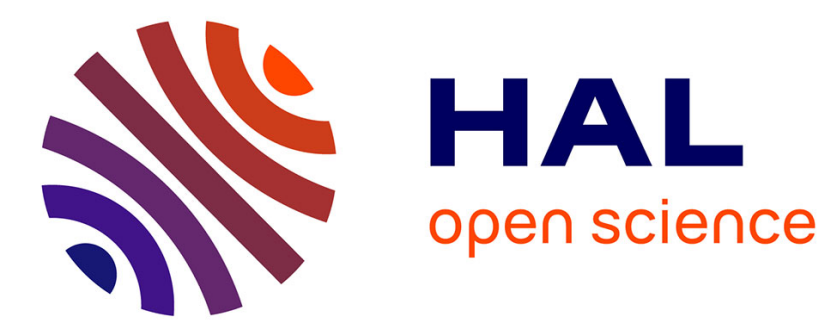

\title{
High-efficiency single-frequency Brillouin fiber laser with a tunable coupling coefficient
}

\author{
Stéphanie Norcia, Robert Frey, Sylvie Tonda-Goldstein, Daniel Dolfi, \\ Jean-Pierre Huignard
}

\section{- To cite this version:}

Stéphanie Norcia, Robert Frey, Sylvie Tonda-Goldstein, Daniel Dolfi, Jean-Pierre Huignard. Highefficiency single-frequency Brillouin fiber laser with a tunable coupling coefficient. Journal of the Optical Society of America B, 2004, 21 (8), pp.1424-1430. hal-00867060

\section{HAL Id: hal-00867060 \\ https://hal-iogs.archives-ouvertes.fr/hal-00867060}

Submitted on 27 Sep 2013

HAL is a multi-disciplinary open access archive for the deposit and dissemination of scientific research documents, whether they are published or not. The documents may come from teaching and research institutions in France or abroad, or from public or private research centers.
L'archive ouverte pluridisciplinaire HAL, est destinée au dépôt et à la diffusion de documents scientifiques de niveau recherche, publiés ou non, émanant des établissements d'enseignement et de recherche français ou étrangers, des laboratoires publics ou privés. 


\title{
High-efficiency single-frequency Brillouin fiber laser with a tunable coupling coefficient
}

\author{
Stéphanie Norcia \\ Thales Research \& Technology, Domaine de Corbeville, 91404 Orsay Cedex, France \\ Robert Frey \\ Laboratoire Charles Fabry de l'Institut d'Optique, Centre Scientifique Paris-Sud, Bâtiment 503, 91403 Orsay Cedex, \\ France \\ Sylvie Tonda-Goldstein, Daniel Dolfi, and Jean-Pierre Huignard \\ Thales Research \& Technology, Domaine de Corbeville, 91404 Orsay Cedex, France \\ Received September 9, 2003; revised March 26, 2004; accepted April 1, 2004

\begin{abstract}
taking pump depletion into account. The output pump and Stokes intensities are calculated as functions of the cavity coupling coefficient and of the input pump intensity. Lasing threshold and pump-to-Stokes conversion efficiency are predicted. Furthermore, we demonstrate good agreement between model results and measurements. Applications to the improvement of optoelectronic links for radio-frequency signals by use of stimulated Brillouin scattering fiber lasers are also presented. (C) 2004 Optical Society of America

OCIS codes: $060.2320,060.4370,140.3600,290.5900$.
\end{abstract} \\ We theoretically analyze a high-efficiency single-frequency Brillouin all-fiber ring laser at $1.5 \mu \mathrm{m}$ wavelength,
}

\section{INTRODUCTION}

Stimulated Brillouin Scattering (SBS) is one of the dominant nonlinear processes that can occur in optical fibers; it can significantly limit the performance of optical transmission systems. ${ }^{1}$ However, SBS can be used in several applications, such as Brillouin fiber lasers, ${ }^{2-4}$ Brillouin optical amplifiers, ${ }^{5,6} \mathrm{SBS}$ fiber sensors, ${ }^{7}$ and optical processing of radio-frequency (rf) signals. ${ }^{8-11}$

In optical fibers, SBS (described in Ref. 12, pp. 355357) gives rise to a backscattered beam, the Stokes wave, which is frequency downshifted by $\nu_{B} \approx 11 \mathrm{GHz}$ from the pump optical frequency (for $\lambda_{\text {pump }} \cong 1.5 \mu \mathrm{m}$ ). Generation of the Stokes wave occurs within a frequency bandwidth $\Delta \nu_{B}$ of $\sim 10 \mathrm{MHz} .^{12}$

SBS begins once the pump power reaches the Brillouin threshold. This threshold increases as the fiber length decreases. Using long optical fibers then enhances SBS efficiency. Furthermore, reinjection of the Stokes wave at the fiber output end, in the reverse pump direction, drastically reduces the SBS threshold. However, in a long fiber ring, several longitudinal Stokes modes can oscillate within the $10-\mathrm{MHz}$-wide SBS gain curve. Such multimode behavior of the circulating Stokes wave can be detrimental for applications such as optical distribution of analog rf signals. ${ }^{13}$

To generate a single-longitudinal-frequency Stokes wave with a low power threshold, it is possible to use a short fiber ring laser ${ }^{14}$ that is simultaneously resonant for pump and Stokes waves (doubly resonant cavity). The ring is made resonant for the pump wave's frequency, thus drastically reducing the SBS threshold in the short optical fiber. As the resonator length is chosen to corre- spond to approximately the inverse of the SBS gain bandwidth, only one single-longitudinal-mode Stokes wave can oscillate. In this paper we theoretically analyze the behavior of this single-frequency Brillouin fiber laser, taking pump depletion into account, and we show that high conversion efficiencies can be obtained at any pump powers by use of a tunable optical coupler.

This paper is organized in the following way: The operation of the doubly resonant SBS laser is described in Section 2. The model used to predict SBS laser performance is presented in Section 3. Section 4 contains detailed analyses and comments on the main results: The behaviors of output pump and Stokes intensities are studied as functions of the cavity coupling coefficient, of the input pump's intensity, and of the linear losses. The lasing threshold and the pump-to-Stokes conversion efficiency are estimated with this model, which also predicts the second-order Stokes (2-Stokes) wave threshold. In Section 5, applications of the efficient single-frequency SBS laser, such as improvement in the quality of optically carried rf signals, are explained.

\section{METHOD OF OPERATION}

The method of operation of an efficient single-frequency doubly resonant all-fiber Brillouin laser is described in Fig. 1. A $2 \times 2$ evanescent mode variable optical coupler is used to close the ring cavity with variable coupling coefficient $R$. The coupler is made from two parallel, close optical fibers. One achieves the variable coupling coefficient by changing the spacing between the two parallel optical fibers when a micrometer screw is turned. Ports 


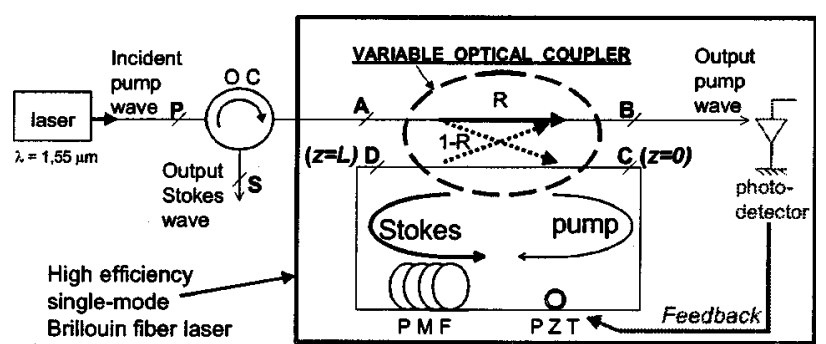

Fig. 1. Efficient single-frequency Brillouin fiber laser.

$\mathrm{C}$ and D of the variable optical coupler are pigtailed to both extremities of a short (20-m-long) polarizationmaintaining optical fiber (PMF). It is a bow-tie-type fiber with an $\sim 5$ - $\mu \mathrm{m}$ core radius, which provides a Brillouin frequency shift $\nu_{B}=10.925 \mathrm{GHz}$. The PMF's linear attenuation coefficient is $\alpha \approx 2.9 \times 10^{-3} \mathrm{~m}^{-1}$. Part of this fiber is wound around a piezoelectric transducer (PZT) to permit control of its length and, therefore, to control the phase accumulated by the pump along the ring. A pump laser beam passes the optical circulator and enters the coupler at port A. It is a 1.5- $\mu \mathrm{m}$ singlefrequency solid-state laser source with a $2-\mathrm{kHz}$ linewidth, followed by an erbium-doped fiber amplifier that provides an output power of as much as $200 \mathrm{~mW}$. The pump is then divided into two parts. One part passes the coupler and goes directly to port $\mathrm{B}$, giving rise to the so-called direct beam. The other part goes to port $\mathrm{C}$ and, after it passes through $\mathrm{PMF}$, enters the coupler at $\mathrm{D}$, giving rise to two beams: One beam is reinjected into the ring and the other one (the so-called transmitted beam) is extracted via port B. The respective intensities of these beams depend on selected coupling coefficient $R$. The values $R=1$ and $R=0$ correspond to no and total cross coupling, respectively. When it is detected at $\mathrm{B}$, the output pump's intensity is used for feedback to control the fiber ring's length to reach a resonant situation for the pump frequency. In this case, constructive interference of the pump waves occurs at port $\mathrm{C}$ and destructive interference between the direct beam and the transmitted beam can be observed in system output B. As this fiber ring is a doubly resonant cavity, no pump light is detected in $\mathrm{B}$ when the direct and the transmitted waves have equal amplitude and therefore interfere completely destructively. For a given level of linear and nonlinear losses experienced by the pump wave in the cavity, these destructive interferences occur only at a given value of $R$. In such a case, the coupling of pump light into the cavity is maximum because all the incident pump power is linearly absorbed or nonlinearly converted to a Stokes wave. As shown hereafter, this situation evidently corresponds to the best achievable pump-to-Stokes conversion efficiency.

When SBS threshold is reached by the pump in the resonator, some of the pump energy is used to generate a Stokes wave traveling in the opposite direction in the ring. Part of the backward-propagating Stokes wave generated along the $\mathrm{PMF}$ is recirculated into the ring via port D; the rest goes out of the ring from port $\mathrm{C}$ to port $\mathrm{A}$ and is available at port $\mathbf{S}$ of the optical circulator. Creation of a Stokes wave induces additional losses for the pump. Maximum conversion efficiency is then obtained by adjusting the value of coupling coefficient $R$ to all the losses of the pump wave in the ring. For input pump powers lower than the Brillouin threshold in the resonator, total losses of the cavity are due only to linear losses, which correspond to the fiber absorption coefficient and to the coupler insertion loss. For input pump powers that permit SBS, a nonlinear loss is added to the cavity as a result of the conversion of a pump wave into a Stokes wave. $^{3}$

Because this resonator is a $20 \mathrm{~m}$-long $(L)$ cavity only, its free spectral range is $\sim 10 \mathrm{MHz}$. The Stokes wave is single frequency because only one cavity mode falls within the 10-MHz-wide SBS gain curve. As demonstrated in Section 4 below, this single-frequency SBS laser reaches high finesse, permitting efficient generation of Stokes power even with low input pump power.

\section{MODEL DESCRIPTION}

Here, the high-efficiency Brillouin fiber laser behavior is analyzed, and pump depletion by SBS is taken into account. The study is made in terms of photon densities. The pump and Stokes photon densities $\left(N_{p}\right.$ and $N_{s}$, respectively) are related to intensities $I_{p}$ and $I_{s}$ (in watts per square meter) by the expression $N_{p, s}=I_{p, s} /\left(\nu_{p, s}\right.$ $\times h$ ), where $\nu_{p, s}$ is the (pump, Stokes) optical frequency and $h$ is the Planck constant.

Setting $N_{p}(z)=n_{p}(z) \exp (-\alpha z)$ and $N_{s}(z)=n_{s}(z)$ $\times \exp (\alpha z)$ to take absorption losses of the fiber into account, we find that Eqs. (1) and (2) describe the coupled variation of the reduced pump and Stokes photon densities $n_{p}$ and $n_{s}$ along the fiber ${ }^{15}$ of propagation axis $z$ for SBS gain $g$ :

$$
\begin{aligned}
& \frac{\mathrm{d} n_{s}(z)}{\mathrm{d} z}=-g n_{s}(z) n_{p}(z) \exp (-\alpha z), \\
& \frac{\mathrm{d} n_{p}(z)}{\mathrm{d} z}=-g n_{s}(z) n_{p}(z) \exp (\alpha z) .
\end{aligned}
$$

For the small absorption losses of our fiber $(\alpha z \ll 1)$ the solution of coupled Eqs. (1) and (2) is expressed as

$$
\begin{aligned}
& n_{s}(z)=\frac{n_{s 0}\left(n_{p 0}-n_{s 0}\right) \exp \left[-g z\left(n_{p 0}-n_{s 0}\right)\right]}{n_{p 0}-n_{s 0} \exp \left[-g z\left(n_{p 0}-n_{s 0}\right)\right]}, \\
& n_{p}(z)=n_{s}(z)+n_{p 0}-n_{s 0},
\end{aligned}
$$

where $n_{p 0}=n_{p}(0)=N_{p}(0)$ and $n_{s 0}=n_{s}(0)=N_{s}(0)$. Ports $\mathrm{C}$ and $\mathrm{D}$ are located at the $z=0$ and $z=L$ extremities, respectively, of the PMF (Fig. 1).

A second set of equations [Eqs. (5) and (6) below] describes the condition for which the pump is resonant in the fiber cavity (constructive interference at port $\mathrm{C}$ and destructive interference at port $\mathrm{B}$, respectively). The pump's photon density at ports B and C can be written as a sum of three terms: the first term corresponds to the input pump's photon density; the second term, to the pump's photon density at port $\mathrm{D}$; and the third term, to the interference between these two contributions. In the same manner, Eqs. (7) and (8) relate transmission of the Stokes wave across the coupler:

$$
n_{p}(0)=d N_{p}{ }^{i}+c n_{p}(L)+2\left[d c N_{p}{ }^{i} n_{p}(L)\right]^{1 / 2},
$$




$$
\begin{aligned}
N_{p}{ }^{t} & =a N_{p}{ }^{i}+b n_{p}(L)-2\left[a b N_{p}{ }^{i} n_{p}(L)\right]^{1 / 2}, \\
N_{s}{ }^{t} & =d n_{s}(0), \\
N_{s}(L) & =c n_{s}(0) .
\end{aligned}
$$

The coupler and fiber losses are introduced in the resonance conditions, so coefficients $a=R\left(1-\alpha_{\mathrm{cpl}}\right), b=(1$ $-R)\left(1-\alpha_{\mathrm{cpl}}\right)\left(1-\alpha_{f}\right), \quad c=R\left(1-\alpha_{\mathrm{cpl}}\right)\left(1-\alpha_{f}\right)$, and $d=(1-R)\left(1-\alpha_{\text {cpl }}\right)$ are related to coupling coefficient $R$, to variable coupler insertion losses $\alpha_{\text {cpl }}$, and to PMF linear absorption losses $\alpha_{f}$, when pigtail losses are taken into account. In these equations $N_{p}{ }^{i}$ is the density of input photons (port A, Fig. 1), $N_{p}{ }^{t}$ is the density of output pump photons (port B), and $N_{s}{ }^{t}$ is the density of output Stokes photons (port A).

From Eq. (5) it is possible to obtain $n_{p}(L)$ as a function of $n_{p 0}$ and $N_{p}{ }^{i}$ :

$$
n_{p}(L)=\frac{\left(\sqrt{n_{p 0}}-\sqrt{d N_{p}^{i}}\right)^{2}}{c} .
$$

Combining Eqs. (8) and (9) in expression (4) at $z=L$ (i.e., at port D) yields the following equation, which shows that a nonlinear function $f\left(n_{p 0}\right)$ links photon densities $n_{p 0}$ and $n_{s 0}$ at port C:

$$
n_{s 0}=\frac{c n_{p 0}-\left(\sqrt{n_{p 0}}-\sqrt{d N_{p}^{i}}\right)^{2}}{c(1-c)}=f\left(n_{p 0}\right) .
$$

Injecting Eq. (8) into Eq. (3) at $z=L$ provides the following expression, which depends only on the photons densities at port $\mathrm{C}$ :

$$
\exp \left[-g L\left(n_{p 0}-n_{s 0}\right)\right]=\frac{c n_{p 0}}{\left[n_{s 0}(c-1)+n_{p 0}\right]} .
$$

As $n_{s 0}=f\left(n_{p 0}\right)$, Eq. (11) can be written as

$$
G\left(n_{p 0}\right)=H\left(n_{p 0}\right),
$$

where

$$
G\left(n_{p 0}\right)=\exp \left\{-g L\left[n_{p 0}-f\left(n_{p 0}\right)\right]\right\}
$$

(a)

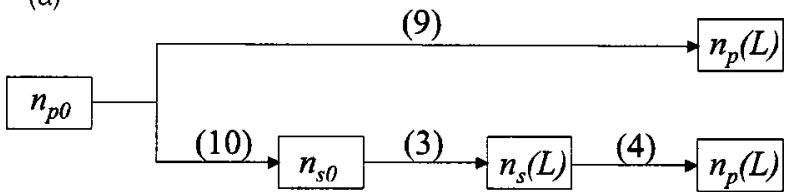

(b)

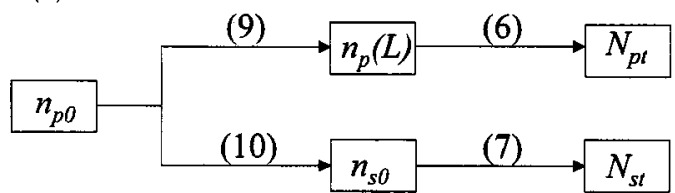

Fig. 2. Resolution method for determination of (a) $n_{p 0}$ and (b) $N_{p}{ }^{t}$ and $N_{s}{ }^{t}$.

$$
H\left(n_{p 0}\right)=\frac{c n_{p 0}}{\left[f\left(n_{p 0}\right)(c-1)+n_{p 0}\right]} .
$$

Figure 2 summarizes the method used in the research reported in what follows to calculate the photon densities (i.e., output) transmitted by the laser. Resolution of Eq. (12) leads to multiple solutions $n_{p 0}$. To find the correct value of $n_{p 0}$ we calculate an intermediate photon density, $n_{p}(L)$, in two different ways, as illustrated in Fig. 2(a). The correct value of $n_{p 0}$ is that which gives equal results for $n_{p}(L)$ calculated by the two ways. Once the correct value of $n_{p 0}$ is determined, the transmitted pump and Stokes photon densities $N_{p}{ }^{t}$ and $N_{s}{ }^{t}$ can finally be deduced simply by solution of Eqs. (9) and (6) and then of Eqs. (10) and (7), as described in Fig. 2(b):

$$
\begin{aligned}
N_{s}{ }^{t}= & \mathrm{d} \frac{c n_{\mathrm{p} 0}-\left(\sqrt{n_{p 0}}-\sqrt{d N_{p}}\right)^{2}}{c(1-c)}, \\
N_{p}{ }^{t}= & \left\{\left[a N_{p}^{i}\right]^{1 / 2}\right. \\
& \left.-\left[b \frac{\left(\sqrt{n_{p 0}}-\sqrt{d N_{p}^{i}}\right)^{2}}{c}\right]^{1 / 2}\right\}^{2} .
\end{aligned}
$$

Equations (15) and (16) give the expressions for $N_{p}{ }^{t}$ and $N_{s}{ }^{t}$ as functions of $n_{p 0}$ and $N_{p}{ }^{i}$. These calculations have been made for several values of $N_{p}{ }^{i}, R, \alpha_{\mathrm{cpl}}$, and $\alpha_{f}$. A few selected results are presented and discussed in Section 4 below.

For Stokes powers that exceed the SBS threshold of the cavity, another corresponding Stokes wave can be generated, the so-called a 2-Stokes wave. The 2-Stokes wave is downshifted from the pump's optical frequency by 2 $\times \nu_{B} \cong 22 \mathrm{GHz}$. It copropagates with the pump wave and then appears at port $B$ of the variable optical coupler. As it was not the aim of this analysis to determine the exact behavior of the 2-Stokes wave, we did not calculate it in the model. This calculation would require solving a more-complex system of three coupled equations instead of the two coupled Eqs. (1) and (2). The equations for the 2-Stokes wave's behavior require taking into account depletion of the first-order Stokes wave. Here we restrict our analysis to first-order Stokes generation. We evaluate the 2-Stokes threshold only, to determine that the Brillouin laser can operate without generating the 2-Stokes component. The variation in photon density of the 2-Stokes wave along the fiber is given by

$$
\frac{\mathrm{d} n_{2 s}(z)}{\mathrm{d} z}=+g n_{2 s}(z) n_{s}(z),
$$

where $n_{2 s}(z)$ is the photon density of the 2-Stokes wave. Taking fiber and coupler losses into account, we find that the oscillation threshold for the 2-Stokes wave in the ring is reached when

$$
c \exp \left\{g\left[\int_{0}^{L} n_{s}(z) \mathrm{d} z\right] L\right\}=1 .
$$

Discussions in Section 4 below prove that the 2-Stokes wave can be avoided in practical setups simply by careful 
adjustment of the value of $R$ for a maximum pump energy conversion to a Stokes wave.

\section{RESULTS AND DISCUSSION}

For easier understanding of orders of magnitude, in this entire section the input pump energy is given in terms of input pump optical power $P_{\text {in }}\left(=N_{p}{ }^{i} \times h \times \nu \times A_{\text {eff }}\right)$, which is directly related to input pump's photon density $N_{p}{ }^{i}$, where $A_{\text {eff }}$ is the effective core area of the PMF. All the calculations were performed with $A_{\text {eff }} \approx 78.5 \mu \mathrm{m}^{2}$ and a SBS gain of $g \approx 5 \times 10^{-11} \mathrm{~m} / \mathrm{W}$ (that is to say, $g$ $\approx 6.63 \times 10^{-30} \mathrm{~m} \mathrm{~s}^{-1}$ ).

Figure 3 illustrates the calculated behavior of the output power as a function of coupling coefficient $R$. It also shows the influence of linear losses of the cavity. The pump power at port B and the Stokes power at port A are normalized to input pump power $P_{\text {in }}$ (port A). For the conditions of Fig. 3 this input power was chosen to be $P_{\text {in }}=150 \mathrm{~mW}$. In a cavity that exhibits no losses, the output pump power presents one extinction for $R \sim 0.26$ and the maximum output Stokes power occurs for the same value of $R$. In this perfect case, $N_{p t}+N_{s t}=N_{p i}$ for each value of $R$. In particular, the maximum output Stokes power is equal to $P_{\text {in }}$, which means a $100 \%$ pumpto-Stokes conversion efficiency. In a cavity with linear losses ( $\alpha_{\mathrm{cpl}}=0.10$ and $\alpha_{f}=0.12$, which correspond to the experimental characteristics of the setup where $\alpha_{\mathrm{cpl}}$ is determined by measurement of ratio between the sum of the output optical powers and the sum of the input optical powers, the extreme values of the output powers are not obtained exactly for the same value of $R$ : Minimum pump power is reached for $R \sim 0.31$ and maximum Stokes power occurs for $R \sim 0.32$. Linear losses also limit the laser efficiency: The maximum output Stokes power is calculated to be $\sim 68 \%$ of $P_{\text {in }}$. The general behavior of the transmitted intensities proves that, for a given value of input pump power, one can choose the laser output pump and Stokes powers (related to $N_{p}{ }^{t}$ and $N_{s}{ }^{t}$ of Section 3) by simply tuning coupling coefficient $R$.

Figure 4 presents the normalized output powers (ratio between the output powers and $P_{\text {in }}$ ) as a function of coupling coefficient $R$ for several values of input pump power $P_{\text {in }}$ (port A). (Values of linear losses are fixed at $\alpha_{\mathrm{cpl}}$ $=0.10$ and $\alpha_{f}=0.12$.) The value of $R$ that yields the extrema of output powers increases as the input pump power decreases. $\quad R \sim 0.25$ when $P_{\text {in }} \sim 190 \mathrm{~mW}$, and $R$ $\sim 0.8$ when $P_{\text {in }} \sim 10 \mathrm{~mW}$. Indeed, the Stokes power generated for one cavity round trip (and consequently the total losses for the pump in the ring) decreases with decreasing values of $P_{\text {in }}$. Let us note that the effective cavity finesse including nonlinear losses can then easily be controlled by tuning of either cavity coupling coefficient $R$ or cavity input pump power $P_{\text {in }}$. In other words, the maximum conversion efficiency of pump energy into Stokes energy occurs for a value of $R$ that depends strongly on the input pump's power in the cavity. The measured maximum output Stokes power is $\sim 72 \%$ of $P_{\text {in }}$ for $P_{\text {in }} \approx 190 \mathrm{~mW}$. Evidently, even higher conversion efficiencies could be obtained by use of a fiber ring cavity with fewer losses.
The calculated pump-to-Stokes conversion efficiency $\left(N_{s}{ }^{t} / N_{p}{ }^{i}\right)$ is shown in Fig. 5 as a function of $P_{\text {in }}$ for $\alpha_{\mathrm{cpl}}$ $=0.10$ and $\alpha_{f}=0.12$. Experiments were performed ${ }^{13}$ with a cavity that had these values of losses and resulted in a measured threshold of $\sim 4 \mathrm{~mW}$. The corresponding measurements are represented by filled circles in Fig. 5, evidencing good agreement between experimental results and our model. The inset of Fig. 5 shows that a perfect cavity permits $100 \%$ conversion efficiency to be reached with less than $500 \mu \mathrm{W}$ of input pump power. The Brillouin threshold of such a cavity would be only $130 \mu \mathrm{W}$.

Let us compare the SBS threshold of this Brillouin laser $\left(P_{\text {th laser }}=3.8 \mathrm{~mW}\right)$ with the SBS threshold of the

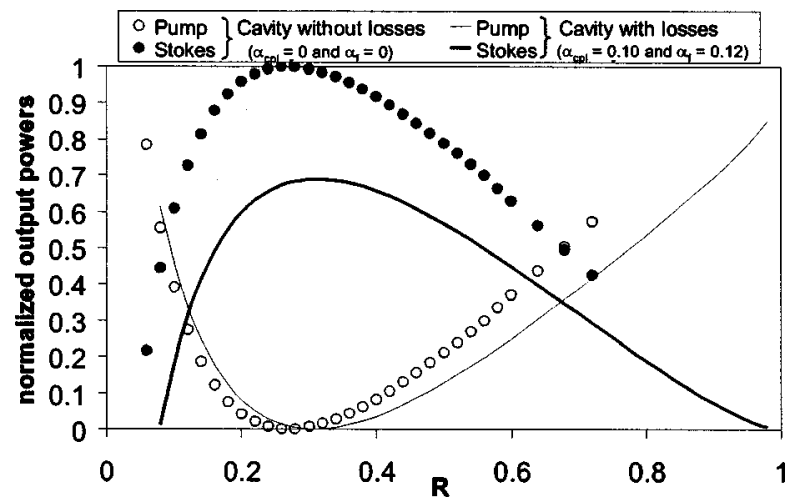

Fig. 3. Normalized pump and Stokes output power as functions of coupling coefficient $R$; $\alpha_{\mathrm{cpl}}$ and $\alpha_{f}$, coupler and fiber losses, respectively.

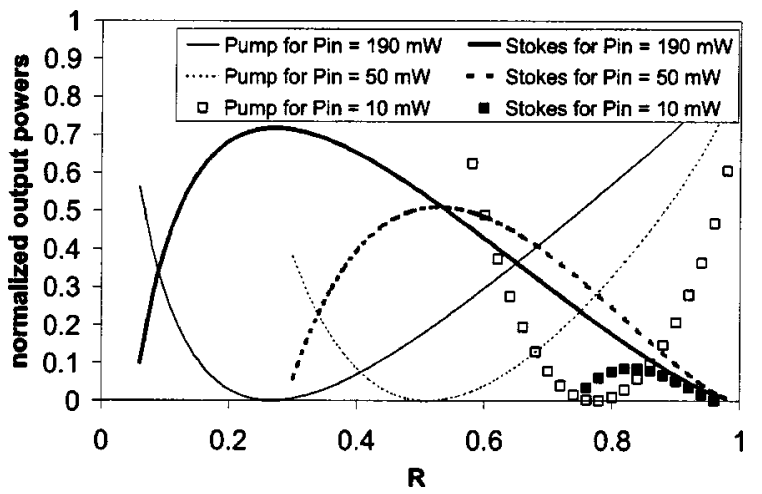

Fig. 4. Normalized output pump and Stokes power as functions of coupling coefficient $R$ for various input pump powers $P_{\text {in }}$.

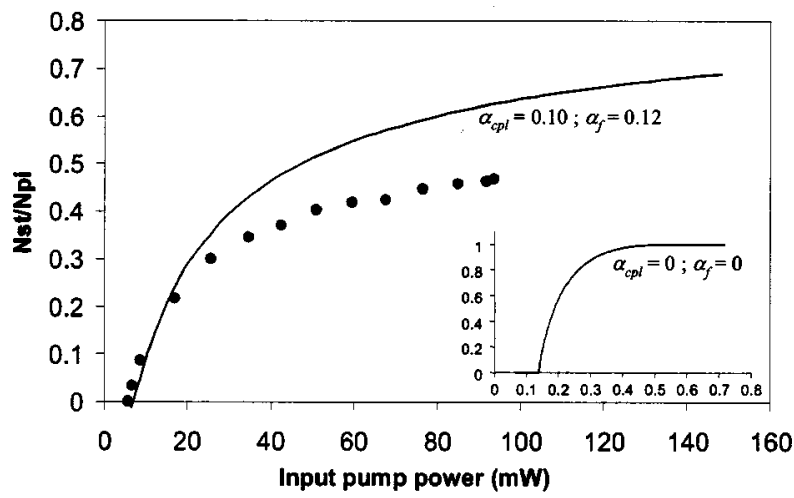

Fig. 5. Pump-to-Stokes conversion efficiency for $\alpha_{\mathrm{cpl}}=0.10$; and $\alpha_{f}=0.12$ and (filled circles) for a cavity without losses. 
same fiber, used in single-pass geometry ( $\left.P_{\text {th_SPass }}\right)$. $P_{\text {th_SPass }}$ can be estimated ${ }^{12}$ as $P_{\text {th_SPass }} \cong 21 A_{\text {eff }} /\left(g L_{\text {eff }}\right)$. The PMF's effective length is $L_{\text {eff }}=[1-\exp (-\alpha L)] / \alpha$, where $\alpha$ denotes the fiber's attenuation coefficient and the PMF's effective core area is $A_{\text {eff }} \approx \pi a^{2}$, where $a$ denotes the fiber core's radius. This estimation gives $P_{\text {th_SPass }}$ $\cong 1.7 \mathrm{~W}$. This result shows that the doubly resonant SBS laser allows the SBS threshold of a 20-m-long PMF to be reduced by more than 500 times. For a singly resonant cavity, the power threshold is

$$
\begin{aligned}
P_{\text {th_SRes }} \cong & \frac{\left\{[(1-R) / R]+\alpha_{\text {cpl }}+\alpha_{f}\right\} A_{\text {eff }}}{g L_{\text {eff }}(1-R)\left(1-\alpha_{\text {cpl }}\right)} \\
& \times\left\{1+\left[R\left(1-\alpha_{\text {cpl }}\right)\left(1-\alpha_{f}\right)\right]^{2}\right\}^{1 / 2},
\end{aligned}
$$

so a reduction by a factor of only $P_{\text {th_SPass }} / P_{\text {th_SRes }} \cong 1.7$ is expected (for $R=0.9, \alpha_{\mathrm{cpl}}=0.10$, and $\alpha_{f}=0.12$ ). Using a doubly resonant cavity enhances intracavity pump intensity, so a supplementary reduction of

$$
\frac{(1-R)\left(1-\alpha_{\mathrm{cpl}}\right)}{\left\{1-\left[R\left(1-\alpha_{\mathrm{cpl}}\right)\left(1-\alpha_{f}\right)\right]^{2}\right\}^{1 / 2}}
$$

is expected, leading to a total threshold reduction of $\sim 250$. This reduction is in good agreement with the experimental value, as the factor of 21 that appears in $P_{\text {th_SPass }}$ is an approximation.

Measured and calculated pump and Stokes output powers (normalized to $P_{\text {in }}$ ) are shown in Fig. 6 for several input pump powers and for $\alpha_{\mathrm{cpl}}=0.10$ and $\alpha_{f}=0.12$. For Stokes powers that exceeded the SBS threshold of the cavity, a 2-Stokes wave was experimentally observed at port $\mathrm{B}$, propagating in the same direction as the pump. In as much as this wave was downshifted by $2 \nu_{B}$ from the pump's optical frequency, it can be discriminated from the pump by an optical spectrum analyzer. As the 2-Stokes wave was not considered in the calculation, measurements differ from the model for the values of $R$ that allow the creation of the 2-Stokes wave. Note that in each case the 2-Stokes wave is obtained for values of $R$ larger than that which gives the maximum first Stokes power, which means that a high-efficiency single-frequency Stokes laser can be obtained at any pump power.

The 2-Stokes wave threshold has been calculated for the following values of losses: (a) $\alpha_{\mathrm{cpl}}=0, \alpha_{f}=0$; (b) $\alpha_{\mathrm{cpl}}=0, \quad \alpha_{f}=0.06 ; \quad(\mathrm{c}) \quad \alpha_{\mathrm{cpl}}=0.05, \quad \alpha_{f}=0$; (d) $\alpha_{\mathrm{cpl}}$ $=0.05, \alpha_{f}=0.06$; (e) $\alpha_{\mathrm{cpl}}=0.10, \alpha_{f}=0.12$. In cases (a)-(d), losses increase (which consequently decreases the generation of first Stokes waves), and the 2-Stokes wave's threshold increases to (a) $800 \mu \mathrm{W}$, (b) $1 \mathrm{~mW}$, (c) $1.7 \mathrm{~mW}$, and (d) $4.5 \mathrm{~mW}$. In case (e) of the experimental setup the 2-Stokes wave threshold is reached for $P_{\text {in }} \geqslant 21.5 \mathrm{~mW}$. This observation is in good agreement with measurements of Fig. 6 that show that no 2-Stokes wave is observed for $P_{\text {in }}=20 \mathrm{~mW}$.

To illustrate that this efficient SBS laser can avoid generating 2-Stokes waves, the values of $R$ that correspond to the 2-Stokes threshold ( $R_{2 s}{ }^{\text {th }}$ ) can be compard with the value that gives minimum output pump power $\left(R_{p}{ }^{\min }\right)$ and maximum pump-to-Stokes conversion efficiency $\left(R_{s}^{\max }\right)$. This comparison is shown in Fig. 7 as a function of input pump power $P_{\text {in }}$, for a cavity with experimental
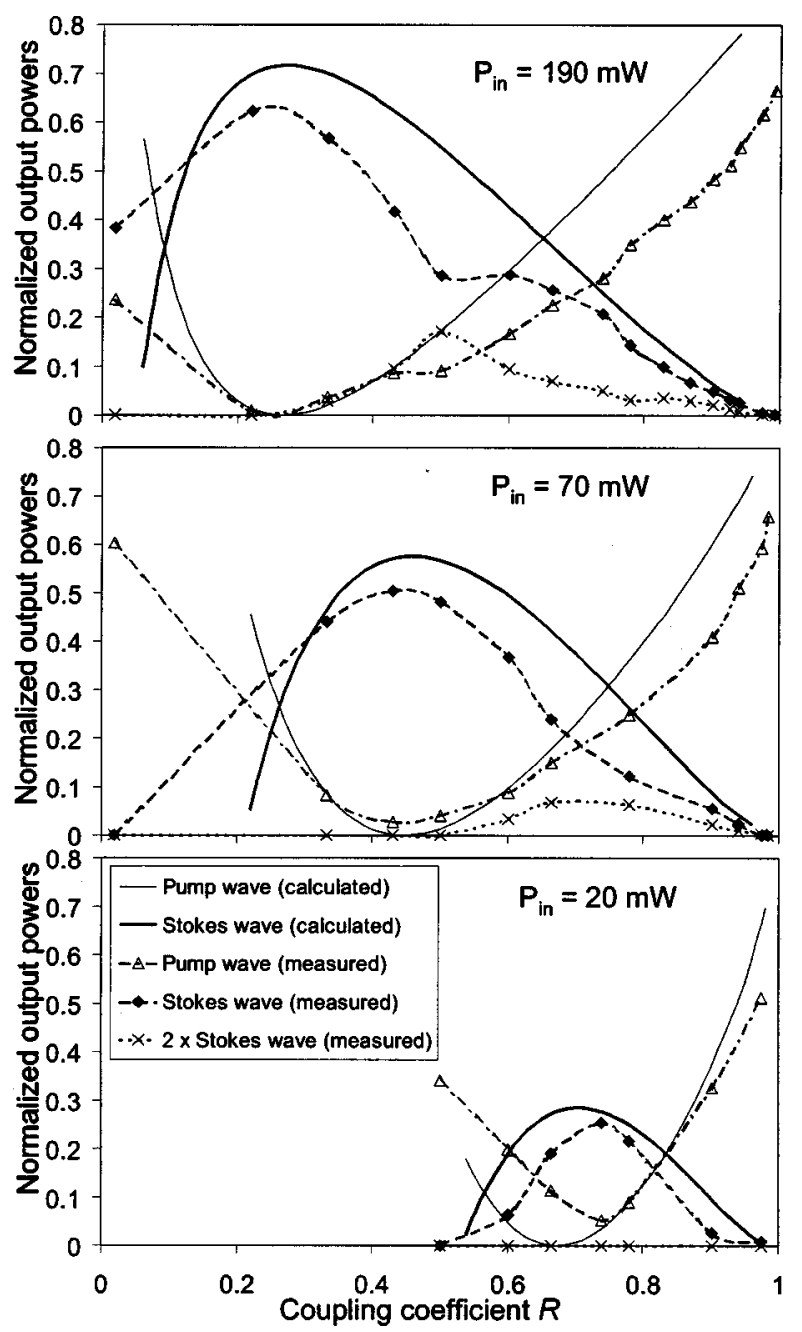

Fig. 6. Normalized output pump, Stokes, and 2-Stokes (2 $\times$ Stokes) powers as a function of coupling coefficient $R$ for various input pump powers $P_{\text {in }}$.

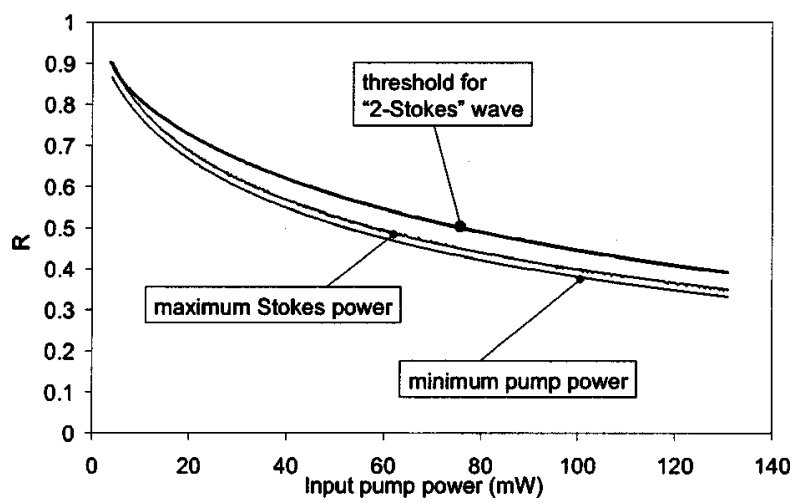

Fig. 7. Values of coupling coefficient $R$ that correspond to extreme output pump and Stokes powers and to the 2-Stoke threshold as a function of input pump power $P_{\text {in }}$.

characteristics $\alpha_{\mathrm{cpl}}=0.10$ and $\alpha_{f}=0.12$. In accordance with observations made in Fig. 6, the value of $R$ that permits the maximum pump-to-Stokes conversion efficiency is below that which permits 2-Stokes generation. Indeed, the calculation shows clearly that $R_{p}{ }^{\min }<R_{s}^{\max }$ $<R_{2 s}^{\text {th }}$, whatever the value of $P_{\text {in }}$. This result permits 
us to conclude that the generation of 2-Stokes waves can be avoided by correct selection of the value of $R$.

\section{APPLICATIONS}

For future radar systems based on optical technologies, optically carried rf transmission and processing of signals will be necessary. Indeed, because conventional optoelectronic modulators permit only limited modulation depths, optically carried rf signals decompose over low-level sidebands and a strong optical carrier. However, too strong an optical component can saturate high-speed detectors. SBS has been demonstrated ${ }^{8-11}$ to be an appropriate mechanism for selectively filtering the excess optical carrier. In particular, recent publications have shown that the single-frequency operation of an efficient Brillouin fiber laser permits as much as $40-\mathrm{dB}$ depletion of the optical carrier ${ }^{16}$ while the quality of the rf signal is maintained in terms of phase noise. ${ }^{13}$ Figure 8 shows the experimental setup that we implemented to demonstrate modulation depth enhancement of optically carried rf signals by the SBS laser. The Stokes wave is injected (in the reverse direction) in to a non-polarizationmaintaining 10-km-long optical fiber carrying a weakly modulated rf signal. The measured optical spectra of the signal are recorded at the optical fiber's input and output. The modulation depth of the input signal is $m_{\text {in }}=0.005$. The signal's optical power is $1 \mathrm{~mW}$ only. Injecting the optical fiber with a $20-\mathrm{mW}$ single-frequency Stokes wave

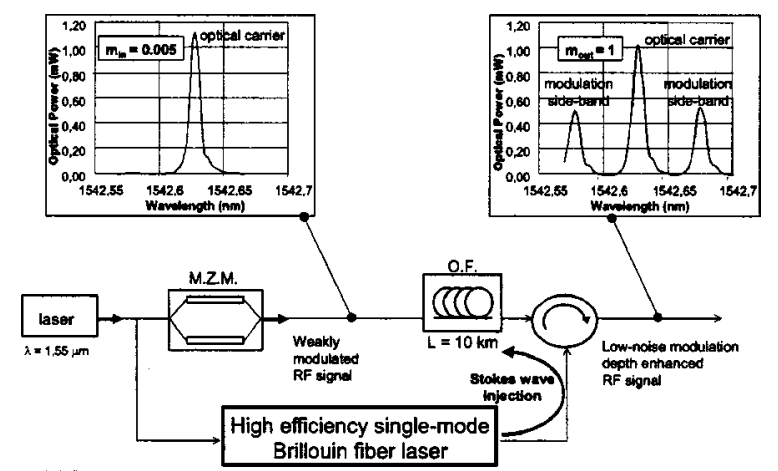

Fig. 8. Use of the SBS laser for modulation depth enhancement of an optically carried rf signal: MZM, Mach-Zehnder modulator; O.F., optical fiber.

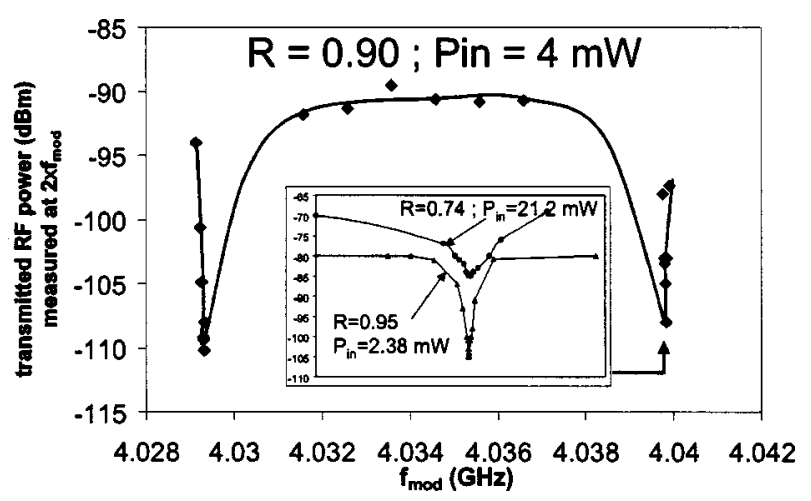

Fig. 9. rf output power of a SBS laser as a function of modulation frequency $f_{\text {mod }}$. from the high-efficiency SBS laser permits 23-dB modulation depth enhancement: The modulation depth of the output signal reaches $m_{\text {out }}=1$.

In addition, the simultaneous resonance of the pump and the Stokes waves in the cavity imposes a strong coherence between the pump laser and the SBS laser. We have also demonstrated ${ }^{13}$ that the single-frequency Brillouin laser can be used for generation of highly pure rf signals, through the beat note between two optical waves, in the range of the frequency offset $\left(\nu_{B}=11 \mathrm{GHz}\right)$ of the pump-to-Stokes waves. Such a beat note could be used for rf local-oscillator generation and distribution for the receive mode of radar systems.

For some rf applications the single-frequency SBS laser can be used as a stand-alone device to control directly the modulation depth of optically carried rf signals. This could be done by injection into the ring of a rf modulated pump wave whose modulation frequency $f_{\text {mod }}$ is not resonant in the cavity (and thus is transmitted to output port B). The last condition has been verified for most values of modulation frequencies: Because of the $10-\mathrm{MHz}$ free spectral range of the cavity, only periodic values of $f_{\bmod }$ are forbidden. As a rf signal is not resonant in the ring, control of the cavity's output pump power (by tuning of $R$, for example) permits control of the output signal's modulation depth. Figure 9 shows the measured output rf power (at port B) as a function of $f_{\text {mod }}$ near $4 \mathrm{GHz}$. For a fixed value of $P_{\text {in }}, R$ is adjusted to reach minimum output power at port B. By tuning $f_{\text {mod }}$ on a range that corresponds to a cavity free spectral range of $\approx-10.513 \mathrm{MHz}$ we could observe two minima in rf output power [at $f_{\min (1)}=4.029320 \mathrm{GHz} \quad$ and $\left.\quad f_{\min (2)}=4.039833 \mathrm{GHz}\right]$, which correspond to coupling of the modulation sidebands to the cavity. The variation of level and shape of the rf resonance [at $f_{\min (2)}=4.039833 \mathrm{GHz}$ ] when cavity finesse $F$ is changed is presented in the inset of Fig. 9. For $P_{\text {in }}$ $=21.2 \mathrm{~mW}$ and $R=0.74$, the contrast in rf signal transmission is $\sim 15 \mathrm{~dB}$ and the resonance width is $\sim 170 \mathrm{kHz}$. For $P_{\text {in }}=2.38 \mathrm{~mW}$ and $R=0.95$, the contrast in rf signal transmission reaches $25 \mathrm{~dB}$ and the resonance width is much narrower, $10 \mathrm{kHz}$ only, which shows that the useful rf range is complete within a factor of $10^{3}$.

The performance of lasers in terms of intensity noise is characterized by the spectral density of the lasers' intensity fluctuations. The transformation of pump intensity fluctuations into Stokes intensity fluctuations in a SBS laser has been demonstrated ${ }^{17,18}$ to be proportional to $\left|\ln \left(R^{2}\right)\right|: \quad$ It then decreases when the finesse of the cavity increases. In particular, for a SBS laser operating high above threshold and with a high value of $R$, the Stokes wave was found to be less noisy than the pump wave over more than two decades. In this paper we have proved that the properties of the SBS laser (good stability because of feedback, the possibility of operating easily above threshold, and the possibility of reaching high-cavity quality values) could facilitate intensity noise filtering of laser sources.

\section{CONCLUSIONS}

In conclusion, we have theoretically analyzed an efficient single-frequency $1.5-\mu \mathrm{m}$-wavelength Brillouin all-fiber 
ring laser, taking pump depletion into account. The behaviors of the output pump and the Stokes intensities were calculated as functions of the cavity coupling coefficient, of the input pump intensity, and of the losses. High conversion efficiencies of pump-to-Stokes power can be obtained because of the presence of a tunable optical coupler. Good agreement between model results and measurements was observed. In addition, the specific properties of this laser should be useful in some future radio-frequency applications.

\section{ACKNOWLEDGMENTS}

We acknowledge contributions to phase noise measurements by T. Merlet, S. Blanc (Thales Air Defense), J. Chazelas, and L. Ménager (Thales Airborne Systems). We thank the Délégation Générale de L'Armement/service des Stratégies Techniques et des Technologies Communes for partial support. We are also grateful to S. Randoux and J. Zemmouri (Centre d'Etudes et de Recherches Lasers et Applications, Université de Lille, France) for fruitful discussions about phase and intensity noise transfer in Brillouin lasers.

\section{REFERENCES}

1. D. Cotter, "Stimulated Brillouin scattering in monomode optical fiber," J. Opt. Commun. 4, 10-19 (1983).

2. L. F. Stokes, M. Chodorow, and H. J. Shaw, "All-fiber stimulated Brillouin ring laser with submilliwatt pump threshold," Opt. Lett. 7, 509-511 (1982).

3. P. Bayvel and I. P. Giles, "Evaluation of performance parameters of single-mode all-fiber Brillouin ring laser," Opt. Lett. 14, 581-583 (1989).

4. F. Zarinetchi, S. P. Smith, and S. Ezekiel, "Stimulated Brillouin fiber-optic laser gyroscope," Opt. Lett. 16, 229-231 (1991).
5. N. A. Olsson and J. P. Van Der Ziel, "Characteristics of a semiconductor laser pumped Brillouin amplifier with electronically controlled bandwidth," J. Lightwave Technol. LT-5, 147-153 (1987).

6. R. W. Tkach and A. R. Chraplyvy, "Fiber Brillouin amplifier," Opt. Quantum Electron. 21, S105-S112 (1989).

7. A. Küng, P. A. Nicati, and Ph. A. Robert, "Reciprocal and quasi-reciprocal Brillouin fiber-optic current sensors," IEEE Photonics Technol. Lett. 8, 1680-1682 (1996).

8. K. J. Williams and R. D. Esman, "Stimulated Brillouin scattering for improvement of microwave fiber-optic link efficiency," Electron. Lett. 30, 1965-1966 (1994).

9. A. Loayssa, D. Benito, and M. J. Garde, "Optical carriersuppression technique with a Brillouin-erbium fiber laser," Opt. Lett. 25, 197-199 (2000).

10. S. Tonda-Goldstein, D. Dolfi, J.-P. Huignard, G. Charlet, and J. Chazelas, "Stimulated Brillouin scattering for microwave signal modulation depth increase in optical links," Electron. Lett. 36, 944-946 (2000).

11. T. Tanemura, Y. Takushima, and K. Kikuchi, "Narrowband optical filter, with a variable transmission spectrum, using stimulated Brillouin scattering in optical fiber," Opt. Lett. 27, 1552-1554 (2002).

12. G. P. Agrawal, Nonlinear Fiber Optics, 3rd ed. (Academic, San Diego, Calif., 2001).

13. S. Norcia, S. Tonda-Goldstein, R. Frey, D. Dolfi, and J.-P. Huignard, "Efficient single-mode Brillouin fiber laser for low noise optical carrier reduction of microwave signals," Opt. Lett. 28, 1888-1890 (2003).

14. L. F. Stokes, M. Chodorow, and H. J. Shaw, "All-single-mode fiber resonator," Opt. Lett. 7, 288-290 (1982).

15. C. L. Tang, "Saturation and spectral characteristics of the Stokes emission in the stimulated Brillouin process," J. Appl. Phys. 37, 2945-2955 (1966).

16. S. Tonda-Goldstein, S. Norcia, D. Dolfi, and J.-P. Huignard, " $40 \mathrm{~dB}$ dynamic enhancement of modulation depth for optically carried microwave signals," Electron. Lett. 39, 790792 (2003).

17. A. Debut, S. Randoux, and J. Zemmouri, "Experimental and theoretical study of linewidth narrowing in Brillouin fiber ring lasers," J. Opt. Soc. Am. B 18, 556-567 (2001).

18. L. Stépien, S. Randoux, and J. Zemmouri, "Intensity noise in Brillouin fiber ring lasers," J. Opt. Soc. Am. B 19, 10551066 (2002). 